\title{
EEG source imaging of brain states using spatiotemporal regression
}

\author{
Anna Custo $^{\mathrm{a}, *}$, Serge Vulliemoz ${ }^{\mathrm{b}, \mathrm{a}}$, Frederic Grouiller ${ }^{\mathrm{c}}$, Dimitri Van De Ville ${ }^{\mathrm{c}, \mathrm{d}}$, Christoph Michel ${ }^{\mathrm{a}}$ \\ a Functional Brain Mapping Lab, University Hospital and Faculty of Medicine, Geneva, Switzerland \\ ${ }^{\mathrm{b}}$ EEG and Epilepsy Unit, Neurology Clinic, University Hospital, Geneva, Switzerland \\ ${ }^{c}$ Department of Radiology and Medical Informatics, University of Geneva, Switzerland \\ d Institute of Bioengineering Ecole Polytechnique Fédérale de Lausanne, Switzerland
}

\section{A R T I C L E I N F O}

\section{Article history:}

Accepted 2 April 2014

Available online 12 April 2014

\section{Keywords:}

EEG source imaging

General Linear Model

Epilepsy

Alpha rhythm

\begin{abstract}
A B S T R A C T
Relating measures of electroencephalography (EEG) back to the underlying sources is a long-standing inverse problem. Here we propose a new method to estimate the EEG sources of identified electrophysiological states that represent spontaneous activity, or are evoked by a stimulus, or caused by disease or disorder. Our method has the unique advantage of seamlessly integrating a statistical significance of the source estimate while efficiently eliminating artifacts (e.g., due to eye blinks, eye movements, bad electrodes).

After determining the electrophysiological states in terms of stable topographies using established methods (e.g.: ICA, PCA, k-means, epoch average), we propose to estimate these states' time courses through spatial regression of a General Linear Model (GLM). These time courses are then used to find EEG sources that have a similar time-course (using temporal regression of a second GLM).

We validate our method using both simulated and experimental data. Simulated data allows us to assess the difference between source maps obtained by the proposed method and those obtained by applying conventional source imaging of the state topographies. Moreover, we use data from 7 epileptic patients ( 9 distinct epileptic foci localized by intracranial EEG) and 2 healthy subjects performing an eyes-open/eyes-closed task to elicit activity in the alpha frequency range.

Our results indicate that the proposed EEG source imaging method accurately localizes the sources for each of the electrical brain states. Furthermore, our method is particularly suited for estimating the sources of EEG resting states or otherwise weak spontaneous activity states, a problem not adequately solved before.
\end{abstract}

(c) 2014 Elsevier Inc. All rights reserved.

\section{Introduction}

The study of brain function has benefited enormously from modern neuroimaging techniques to reveal localization and dynamics of neuronal activity during evoked and spontaneous states. One of the most widely used methodologies to analyze data from functional magnetic resonance imaging (fMRI), is the General Linear Model (GLM) where pre-defined hemodynamic responses are used in a linear regression model and contrasts of interest are evaluated by statistical hypothesis testing (Bandettini et al., 1992; Friston et al., 1995; Kwong et al., 1992; Ogawa et al., 1992). As the fMRI signal is related to neuronal activity via neurovascular coupling, it only provides a (slow) proxy for neuronal activity. Electroencephalography (EEG), on the other hand, directly records the fast changes of current potential related to neuronal activity. Recent advances in high-density recording and 3D source analysis have increased EEG accuracy as a brain imaging method with the inherent advantage of high temporal resolution (Michel and Murray, 2012)

It is fairly natural to conceive the application of conventional GLM analysis as used for fMRI to the Electrical Source Images of the EEG

\footnotetext{
* Corresponding author.

E-mail addresses: anna.custo@unige.ch (A. Custo).
}

(or ESI: with this general term we indicate any method mapping scalp measurements into source space), as some previous papers (Brookes et al., 2004; Kiebel and Friston, 2004; Trujillo-Barreto et al., 2008) and toolboxes propose (such as SPM, www.fil.ion.ucl.ac.uk/spm). These methods follow steps analogous to those performed in fMRI data analysis (i.e., GLM regression, contrast definition, statistical inference) with the difference that the fMRI volumes are substituted with the 3D EEG source images. The GLM regressors used in these methods can originate from known experimental or pathological events forming simple design matrices, like the stimulus onsets, active and passive time windows, or the occurrences of epileptic discharges. The regressors can also be formed from more complex time series like estimated or modeled evoked response waveforms or band-limited frequency power waveforms (Brookes et al., 2004; Trujillo-Barreto et al., 2008). Through these regressors, the interesting portion of the signal is isolated from the rest of the brain activity and the generators contributing to this part of the signal are identified.

Our proposed method starts off with a spatial GLM that uses a set of topographies as regressors, i.e., a set of scalp potential maps. A scalp potential map represents the global momentary state of the brain defined by the sum of all momentary active sources. Many previous multichannel EEG studies have demonstrated that the spatial configuration of the 
potential map very sensitively reflects particular brain states (Lehmann et al., 2009). For example, different event-related potential (ERP) components are characterized by specific map topographies and subtle differences in these topographies can be correlated with differences in stimulus characteristics or processing capacities (Brandeis and Lehmann, 1986; Makeig et al., 1997; Michel and Murray, 2012; Michel et al., 2001). Processing-specific map topographies can even be found in single-sweep ERPs without visible patterns in the waveforms (De Lucia et al., 2007). Similarly, topographic differences in spontaneous band-limited frequency power can be directly related to local variations in attention or to mental diseases or pathologies (Kikuchi et al., 2007; Rihs et al., 2009; Zelmann et al., 2013). Spontaneous activity can also be described by a limited number of distinct potential maps repeating in time (EEG microstates, Lehmann et al., 1998). Finally, pathological activities, such as epileptic discharges are characterized by focus-specific typical topographies (Michel et al., 1999; Scherg et al., 2012).

In the first stage of our method we estimate the time courses of the EEG topographies of interest, which describe the salient brain states characterizing the EEG data acquired. This set of EEG maps acts as a spatial filter of the EEG by selecting only the sources time-correlated with the chosen maps. In fMRI analysis a similar approach was applied to combined EEG-fMRI data (Britz et al., 2010; Musso et al., 2010; Grouiller et al., 2011): the time-courses of given EEG maps were convolved with the hemodynamic response function (HRF) generating correlated BOLD-responses representing the brain areas contributing to the generation of the EEG maps, i.e., belonging to the same brain state. Characteristic BOLD-networks closely resembling known resting-state networks have been found when using this method to convolve typical EEG topographies at rest with the BOLD signal (Britz et al., 2010). Similarly, in a recent combined EEG-fMRI study (Grouiller et al., 2011) the time course of maps characterizing patient-specific epileptic discharges was used as regressor to localize the corresponding BOLD response and the approach proved successful despite the absence of interictal discharges during the data acquisition. These studies confirm that EEG topographies represent neurophysiological states even in the absence of typical EEG or ERP waveform patterns (Britz et al., 2010; de Lucia et al., 2007; Grouiller et al., 2011).

The key of our method is to define the state design matrix: the set of states (i.e., scalp topographies) that explains relevant parts of the data. The identification of the set of significant states leads to the estimation of the corresponding temporal regressors via a GLM procedure. The GLM outcome represents the time courses of the states of interest, i.e., the states explaining the key portion of the scalp measurements. Non-physiological and physiological artifacts, like bad electrodes, eye movements, or cardiac rhythm (extracted, for example, using ICA), can be included in the model in the form of corresponding topographies, thereby "regressing out" these artifacts from the relevant part of the signal, as we demonstrate in our second validation example (see the Mapping the generators of alpha rhythm section).

These temporal regressors are then used by a second GLM that computes their corresponding sources (much like previous methods, e.g.: Ogawa et al., 1992; Friston et al., 1995). Fitting the EEG to estimate the regressors allows us to exploit the temporal dynamics of relevant neurophysiological states, instead of deriving the regressors from preset conditions, such as stimulus onset. On the other hand, the possibility of defining the state design matrix from some decomposition of the scalp measurements (like ICA in Jung et al. (2001); or k-means cluster analysis in Pascual-Marqui et al. (1995)), as well as from prior information or other additional data, leads to the creation of a more flexible fitting model of the EEG data itself.

After describing the method in the following section, we validate it using recordings of epileptic activity of seven patients and recordings of spontaneous alpha rhythms in two healthy subjects. We chose these two datasets as a proof-of-concept because we have complementary evidence about the sources' location. Namely, for the alpha rhythm we analyzed simultaneous EEG-fMRI recordings and compare our EEG-based source maps with the BOLD-based results and with the electrical source image (ESI) of the alpha state map. In the epileptic patients, invasive validation from intracranial EEG or post-operative follow-up was available, in addition to the non-invasive work-up (ESI). We also selected cases with simultaneous EEG-fMRI recordings where focus localization with fMRI is compared with our EEG-based localization method. Furthermore, we simulated an EEG recording and compared the accuracy of our method predictions with the more direct approach of projecting the topographies of interest into source space.

\section{Methods}

\section{Method description}

Multichannel whole-scalp EEG recordings consist of time-series of scalp potential maps, $m(s, t)$, where $s$ represents the electrode position and $t$ represents the time. We assume that the different brain states are defined by different topographies in these series of maps and define them as $M_{i}$, with $i=1: N$, where $N$ is the number of states.

For each time frame $t_{0}$, we assume the following GLM:

$m\left(s, t_{o}\right)=\sum_{i}\left(M_{i} \times T_{i}\left(t_{o}\right)\right)+\epsilon$,

where $\epsilon$ is the residual noise, $M_{i}$ is the topographic map indicating an electrophysiological state, $T_{i}$ is the coefficient to be estimated, and $i$ spans across the number of states we wish to include to sufficiently explain the data. Solving Eq. (1) by least-square regression leads to the estimate of the coefficients time courses, $\hat{T}_{i}(t)$, which are then used as regressors in the second phase of the process (Eq. (2)). $\hat{T}_{i}(t)$ expresses the time course of the brain state $i$ we are computing the sources of, and $t$ is the dummy variable expressing the time dimension. Our algorithm, that we name TESS for Topographic Electrophysiological State Source-imaging, automatically prompts a warning in case any pair of estimated $\hat{T}_{i}(t)$ is (close to) co-linear.

In the second stage of our method we transform the EEG data into source space, also called inverse space. Each EEG time frame in sensor space, $m\left(s, t_{o}\right)$, is transformed into source space, $m_{i n v}\left(v, t_{o}\right)$, using an ESI method (Michel et al., 2004). $m_{i n v}(v, t)$ represents the brain generators of the scalp measurements as a function of solution points $v$ and time $t$. For each position $v_{o}$ we assume:

$m_{\text {inv }}\left(v_{o}, t\right)=\sum_{i}\left(\hat{T}_{i}(t) \times \beta_{i}\left(v_{o}\right)\right)+\eta$,

where $\eta$ is the residual noise, the $T_{i}$ was estimated from Eq. (1) and $\beta_{i}$ is the new coefficient to be estimated. Eq. (2) defines a temporal GLM and its solution is $\hat{\beta}_{i}(v)$, the estimate of $\beta_{i}$ for each solution point $v_{o}$. We performed a non-parametric statistical test of $\hat{\beta}_{i}(v)$ by randomly permuting the estimated $\hat{T}_{i}(t)$ over $t$ and re-computing the betas. After repeating this procedure several times we generated an empirical nulldistribution of the source-maps and computed the Bonferroni corrected z-scores from its tail (see Appendix A for details). The significant estimated betas give us the location and amplitude of the generators of each state $M_{i}$ we initially included in the state design matrix. In the Discussion section we will address some issues related to the design matrix specification (i.e., choosing the right set of states $M_{i}$ ).

In Fig. 1, we illustrate the different steps of the method using data from an epileptic patient of validation 1 (see below). Two state maps were selected corresponding to the average maps of this patient's two types of epileptic spikes ( $M_{1}$ and $M_{2}$, left anterior and left posterior spikes, respectively). The first step of the method (Fig. 1, top row) estimates the time courses, $\hat{T}_{1}$ and $\hat{T}_{2}$, of these two maps. In the second phase (Fig. 1, bottom row), we use these time courses as regressors for the ongoing EEG transformed into source space and estimate $\hat{\beta}_{1}$ and $\hat{\beta}_{2}$ for each voxel. We then select only the z-score corresponding to the statistically significant portion of $\hat{\beta}$. In Fig. 1 


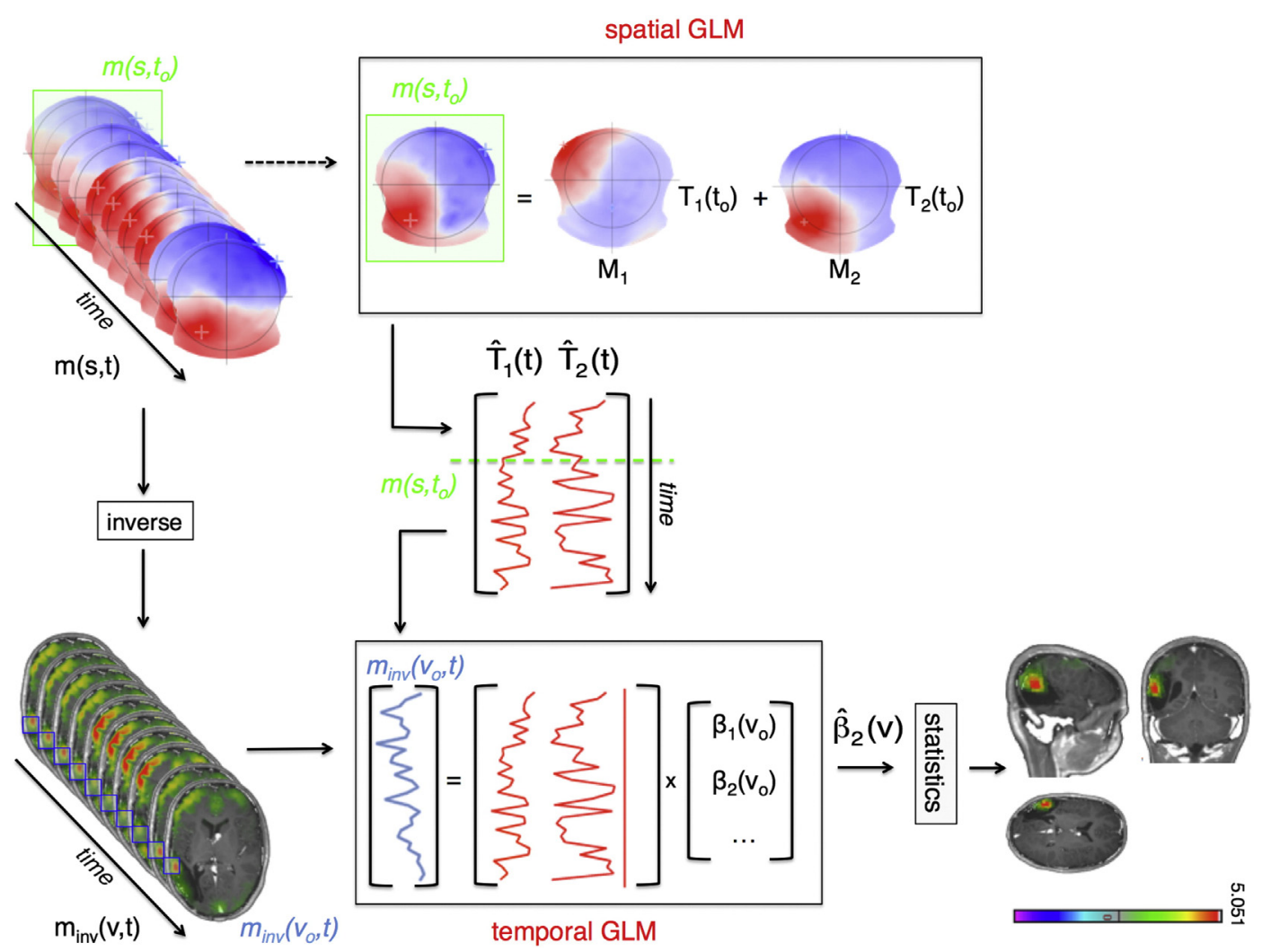

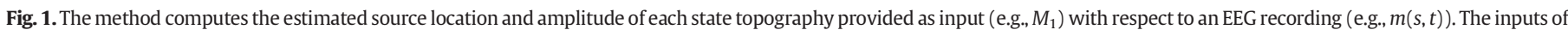

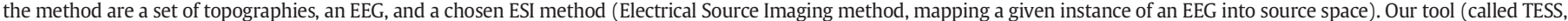

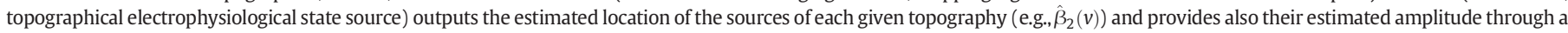

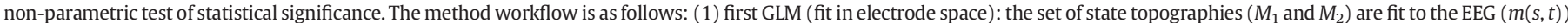

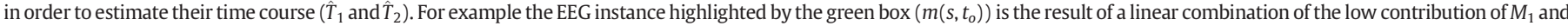

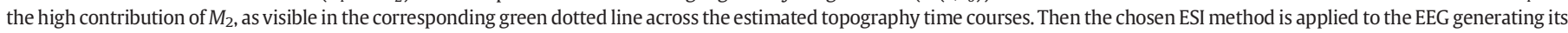

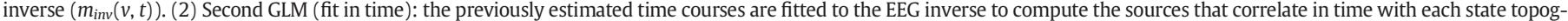

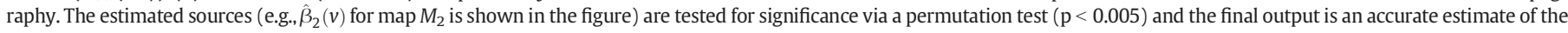
location and amplitude of the generators of each input topography.

bottom right the significant z-score of $\hat{\beta}_{2}$ is overlaid with the patient post-operative anatomical MRI. It shows correct localization of the activity generating the epileptic map $M_{2}$ in the afterwards resected area.

For the ICA performed in some of the EEG pre-processing we use the criterion proposed by Onton et al. (2006): for high resolution EEG, the number of time frames for a good decomposition should be at least $k \cdot(\text { number of channels })^{2}$ with $k=20$. Thus, when we do not have enough time frames (i.e., EEG duration $<22$ min with 256 electrodes), we whiten the signal (PCA) with a number of components $N$ calculated to respect the original criteria $(N=\sqrt{(\text { number of time frames)/ }}$ and $k=2$ ), which usually leads to 20-80 independent components.

In the Appendix A we provide a detailed description of the method in matrix notation.

\section{Validation}

\section{Mapping the source of epileptic activity}

We analyzed 7 patients with drug resistant focal epilepsy from the pre-surgical epilepsy evaluation unit of the Neurology Department of Geneva University Hospital (Switzerland). We acquired highdensity EEG recordings of all patients (Table 1a); in addition, two patients had simultaneous EEG-fMRI (Table 1b). In all cases, validation was obtained from subsequent intracranial EEG in one patient (icEEG) and from surgical resection leading to seizure freedom in the other cases.
The local ethics committee approved the recording of EEG and EEGfMRI and all patients and the parents of pediatric patients gave their written informed consent.

Data acquisition. In 5 patients, EEG was recorded outside the MR scanner (extra-MRI EEG) during 20 min of rest with eyes closed, using a highdensity cap (256 channels, Electrical Geodesics Inc., Eugene, OR).

For 2 patients (S6 and S7) the analysis was performed on the EEG acquired during fMRI recording (intra-MRI EEG). Twenty minutes of rest with eyes closed was recorded using a 64-channel MR-compatible EEG system (Brain Products GmbH, Gilching, Germany). This EEGfMRI dataset was particularly useful for direct comparison of our method with the fMRI result. The same regressors were used for the GLM analysis with the ESI and with the BOLD response (but convolved with the HRF and down-sampled to the fMRI's TR). The functional MRI was acquired using $\mathrm{T}_{2} *$-weighted single-shot gradient-echo echoplanar images with a 3-Tesla MR scanner (TR $=1500 \mathrm{~ms}$, voxel size: $3.75 \times 3.75 \times 5.5 \mathrm{~mm}^{3}, 25$ slices).

\section{Data pre-processing}

Extra-MRI EEG (Table 1a). The EEG recorded outside the MR scanner was corrected for pulse and eye-blink artifacts using Independent Component Analysis (Bell and Sejnowski, 1995). Bad electrodes were interpolated using a 3D spline (Brunet et al., 2011).

Intra-MRI EEG (Table $1 \mathrm{~b}$ ). Gradient and pulse artifacts (i.e., all artifacts time-locked to cardiac activity) were removed from the EEG using average artifact subtraction methods (Allen et al., 1998, 2000; 
Table 1

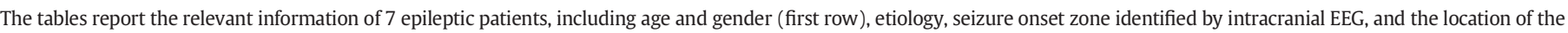

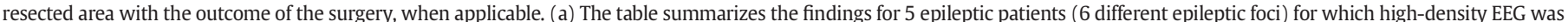

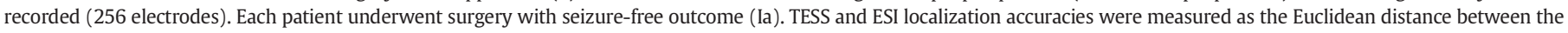

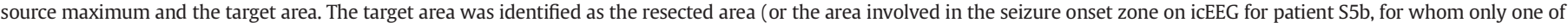

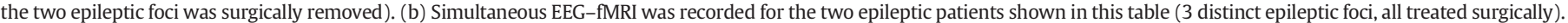

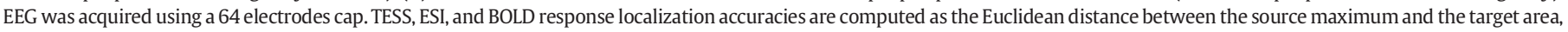

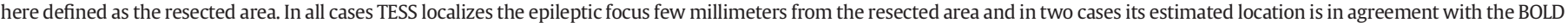
response localization (BOLD results is inconclusive in the third case).

\begin{tabular}{|c|c|c|c|c|c|c|c|}
\hline \multicolumn{8}{|l|}{ Extra-MRI EEG (a) } \\
\hline Patient & \multicolumn{2}{|c|}{ S1 18 y M } & S2 17 y M & S3 11 y M & S4 15 y M & S5a 15 y F & S5B 15 y F \\
\hline Etiology & \multicolumn{2}{|c|}{ Non-lesional } & Hippocampal sclerosis L & Non-lesional & $\begin{array}{l}\text { Low grade tumor L medial } \\
\text { temporal }\end{array}$ & Tuberous sclerosis & Tuberous sclerosis \\
\hline icEEG seizure onset zone & \multicolumn{2}{|c|}{ L basal and polar temporal } & NA & R opercular & NA & L parietotemporal & L anterio-temporal \\
\hline Resection & \multicolumn{2}{|c|}{ LATLR } & LATLR & $\mathrm{R}$ opercular & LATLR & L parietotemporal tuber & NA \\
\hline Outcome & & la & la & la & la & la \\
\hline TESS max distance from target area & \multicolumn{2}{|c|}{$0 \mathrm{~mm}$} & $0 \mathrm{~mm}$ & $0 \mathrm{~mm}$ & $0 \mathrm{~mm}$ & $0 \mathrm{~mm}$ & $0 \mathrm{~mm}$ \\
\hline ESI max distance from target area & \multicolumn{2}{|c|}{$0 \mathrm{~mm}$} & $0 \mathrm{~mm}$ & $95 \mathrm{~mm}$ & $0 \mathrm{~mm}$ & $0 \mathrm{~mm}$ & $0 \mathrm{~mm}$ \\
\hline \multicolumn{8}{|l|}{ Intra-MRI EEG (b) } \\
\hline \multicolumn{2}{|l|}{ Patient } & \multicolumn{2}{|l|}{ S6a 13 y F } & \multicolumn{2}{|l|}{ S6b 13 y F } & \multicolumn{2}{|l|}{ S7 7 y M } \\
\hline & \multicolumn{2}{|c|}{ Tuberous sclerosis } & \multicolumn{2}{|c|}{ Tuberous sclerosis } & \multicolumn{2}{|c|}{ L parieto-temporal gliosis post-bacterial abscess } \\
\hline \multicolumn{2}{|l|}{ icEEG seizure onset zone } & \multicolumn{2}{|c|}{ L prefrontal and opercular tubers } & \multicolumn{2}{|c|}{ L prefrontal and opercular tubers } & \multicolumn{2}{|c|}{ L parieto-temporal } \\
\hline \multicolumn{2}{|l|}{ Resection } & \multicolumn{2}{|c|}{ L prefrontal tuber } & \multicolumn{2}{|c|}{ L frontal tuber } & \multicolumn{2}{|l|}{ L parieto-temporal cortectomy } \\
\hline \multicolumn{2}{|l|}{ Outcome } & \multicolumn{2}{|l|}{ la } & \multicolumn{2}{|l|}{ la } & \multicolumn{2}{|l|}{ la } \\
\hline \multicolumn{2}{|l|}{ TESS max distance from resected area } & \multicolumn{2}{|l|}{$3 \mathrm{~mm}$} & \multicolumn{2}{|l|}{$5 \mathrm{~mm}$} & \multicolumn{2}{|l|}{$5 \mathrm{~mm}$} \\
\hline \multirow{2}{*}{\multicolumn{2}{|c|}{ ESI max distance from resected area }} & \multicolumn{2}{|l|}{$0 \mathrm{~mm}$} & \multicolumn{2}{|l|}{$85 \mathrm{~mm}$} & \multicolumn{2}{|l|}{$0 \mathrm{~mm}$} \\
\hline & & $15 / 0 \mathrm{~mm}^{\mathrm{a}}$ & & / & & $2 \mathrm{~mm}$ & \\
\hline
\end{tabular}

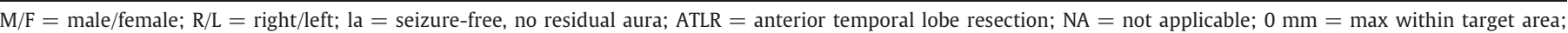
/ = inconclusive.

a Global/local maximum.

Debener et al., 2008). If necessary, residual artifacts were removed with temporal Independent Component Analysis of the EEG using the Infomax approach (Bell and Sejnowski, 1995). Independent components corresponding to pulse artifacts, eye-blink or residual scanner artifacts were identified by visual inspection of ICA components' time courses and topographies and were removed.

Average spike maps. An expert epileptologist marked the spikes and computed the average spike map for each patient's type of spike. The number of spikes recorded varied between 11 and 304 (59.4 \pm 92.9, mean \pm standard deviation). The average maps at half rise (i.e., at the mid-point between the onset of epileptic activity and its apex) were selected as representation of the epileptic map of the patient, i.e., the brain state reflecting the interictal epileptic activity (Lantz et al., 2003). These maps were used for the GLM analysis in the EEG source-space as well as for the convolution with the BOLD response (as described in Grouiller et al., 2011; see below).

Source-space projection. We transformed the average spike maps into source space generating their ESI (see Table 1a-b). For all the projections into source space we used a distributed linear inverse solution based on local autoregressive averages (Grave de Peralta et al., 2004). A simplified realistic head model was used with the solution space restricted to the gray matter of the individual MRI (Brunet et al., 2011).

fMRI. Standard fMRI pre-processing was performed with SPM8 (Wellcome Department of Imaging Neurosciences, University College London, UK) and included: (i) realignment of functional images using rigid-body transformation, (ii) co-registration of functional images with pre- and/or post-operative T1-weighted structural MRI, and (iii) spatial smoothing with an isotropic Gaussian kernel of $6 \mathrm{~mm}$ full width at half-maximum.

fMRI time-series were analyzed with a General Linear Model in which the regressors of interest were derived from the fitting of the epileptic map to the EEG recorded in the MR (Grouiller et al., 2011) Six motion-related parameters derived from the fMRI realignment were included as covariates. For each patient, BOLD signal increases or decreases associated with the epileptic regressor were detected using a significance level of $\mathrm{p}<0.05$ corrected for multiple comparisons across the whole brain using family wise error (FWE) correction. If no significant activation was found, maps were created at a significance level of $\mathrm{p}<0.001$ uncorrected for multiple comparisons.

Performance metrics. Localization accuracy was measured as the Euclidean distance between the source distribution global maximum and the target area (Brodbeck et al., 2011). The target area was identified as the resected area or the area involved in the seizure onset zone on icEEG for patient S5b (the only case in which surgery was not performed).

Our localization metric was chosen to match current clinical practice (based on ESI) and in a sense it is quite strict. When a statistical threshold is available (like for the BOLD signal and TESS) a metric accounting for the extent of the cluster of maximal activity is also meaningful, but it is not comparable to ESI results.

\section{Mapping the generators of alpha rhythm}

We recorded combined EEG-fMRI in two healthy subjects during $5 \mathrm{~min}$ of eyes-open/eyes-closed activity where the subject was instructed to alternate these states every $20 \mathrm{~s}$, resulting in about 5 state transitions. This task is known to induce high alpha power modulations in the occipital cortex while reducing inter-subject variability and frequency bands crosstalk when compared to measurements of rest with only eyes open or closed (De Martino et al., 2010).

Data acquisition. EEG was acquired at $1 \mathrm{kHz}$ using a GES 300MR highdensity EEG system (Electrical Geodesics Inc., Eugene, OR) with a 256 electrode net.

Functional MRI was acquired using a $\mathrm{T} 2{ }^{*}$-weighted single-shot gradient-echo echo-planar images with a 3-Tesla MR scanner (TR $=2000 \mathrm{~ms}$, voxel size: $3 \times 3 \times 3.5 \mathrm{~mm}^{3}$, 34 slices). A highresolution anatomical MR scan was also acquired for each subject (voxel size: $1 \times 1 \times 1 \mathrm{~mm}^{3}$ ). 


\section{Data pre-processing}

$E E G$. We removed gradient and pulse artifacts from the intra-MRI EEG using average artifact subtraction methods (Allen et al., 1998, 2000; Debener et al., 2008). The data was then down sampled to $125 \mathrm{~Hz}$ and bad electrodes were interpolated using a 3D spline algorithm (Brunet et al., 2011). We band-pass filtered this EEG between 8 and $12 \mathrm{~Hz}$ to isolate the alpha rhythm. For additional signal stability we removed few time frames dominated by artifacts induced by eye movement between state transitions (eyes open/closed). The same distributed linear inverse solution using the individual MRI for constructing the head model was used.

The topographies related to eye-blink and physiological activity were selected with temporal ICA of the EEG using the Infomax approach (Bell and Sejnowski, 1995) and the decomposition criterion proposed by Onton et al. (2006). We identified 9 artifact topographies for each subject and we included them in the set of state maps forming TESS' design matrix.

fMRI. MR functional data were analyzed as described above. The regressor associated with the alpha rhythm was computed as described below. BOLD signal decreases were then expressed as equivalent z-scores and corrected for multiple comparisons (FWE). We used different correction methods (FWE for BOLD signal, Bonferroni for TESS) considering the number of observations in each statistical test (in the order of $800,000-900,000$ for BOLD data and 4000-5000 for TESS). We opted for this choice since applying Bonferroni for BOLD results would be clearly over-conservative, while FWE for TESS would require non-trivial adaptation of Gaussian random field theory to the (nonCartesian) grid of solution points.

Regressors. The alpha state map used for the analysis was defined as the EEG independent component best representing the occipital alpha rhythm in terms of topographical distribution and its time course (De Martino et al., 2010). For the EEG analysis through TESS we added 9 independent components to the state design matrix to capture the eye blinking artifacts and physiological activity of the brain at rest, as it has been proposed for fMRI analysis in Chaudhary et al. (2012).

The power of time course of the same EEG alpha state map was used as a regressor for the BOLD data analysis, after convolving it with the HRF and down sampling it to the fMRI sampling rate (De Martino et al., 2010). Motion-related regressors were also added to the BOLD design matrix.

\section{Simulated source}

In order to quantify the performance difference between our method and the conventional direct application of electric source imaging to the state topographies, we simulated a random time series of an occipital source (Fig. 4(A), in the green box) and uncorrelated background sources. We computed the corresponding EEG scalp measurements with additional white Gaussian noise and applied an ESI method (LAURA, Grave de Peralta et al., 2004) to a noisy version of the true topography (in Fig. 4: the noisy (b) and true (a) scalp maps, and in Fig. 4(B), red box, the ESI of map (b)). Then we used the noisy occipital map and the background state map as well as the simulated EEG as input to our new method to estimate the corresponding sources.

\section{Results}

\section{Epilepsy source imaging}

The results summarized in Table 1a-b indicate the high accuracy of the new localization method to identify the generators of the individual-specific epileptic map. In the last rows of the tables we show the Euclidean distance between TESS/ESI/BOLD maximum and the target area. In $5 / 7$ patients ( 6 different epileptic foci) the generator maxima, as well as its significant extent, fell within the target area (Table $1 \mathrm{a}$ ). In the other $2 / 7$ patients ( 3 distinct epileptic foci) the generator maximum was localized within $5 \mathrm{~mm}$ of the target/resected area
(Table 1b). In these two cases, TESS localization is in agreement with the BOLD data and ESI in 2/3 foci (in the third case BOLD results are inconclusive and ESI global maximum falls $85 \mathrm{~mm}$ from the resected area) (Table 1b last two rows). We chose these 2 patients to test our method in the case of noisy intra-MRI EEG recordings with fewer electrodes (64 instead of 256) and rare spike events, and in order to compare our method's performance with BOLD's as well as icEEG's results.

In Fig. 2 we show the localization of the epileptic foci for one patient (S5). This patient has tuberous sclerosis, a genetic form of multifocal epilepsy with multiple abnormalities of cortical development. He had two independent interictal epileptogenic foci confirmed by intracranial EEG using several subdural grids and strips. The most active interictal focus, and corresponding to the seizure onset zone, was located in the parietotemporal tuber, which was resected with a seizure free outcome. In Fig. 2 we show the location of the icEEG electrodes that recorded epileptic activity continuously (group 1), less frequently (group 2), and rarely (group 3). We display the localization of two epileptic maps corresponding to two distinct spikes at 50\% rising phase. Both solutions were overlaid on the post-operative anatomical MRI. Even the less frequent epileptic activity (Fig. 2 right panel) was successfully localized, as also confirmed by icEEG. The most active interictal source has been shown to be concordant with the epileptogenic zone in most patients (Mégevand et al., 2014), even in cases of multifocal spiking such as tuberous sclerosis (Kargiotis et al., accepted for publication), supporting the clinical interest of our method.

\section{Alpha rhythm source imaging}

In Fig. 3 we show the areas involved during alpha modulation in both subjects (green and yellow boxes) and the EEG topography of the alpha state. As expected, we found primarily activity in the occipital cortex (primary visual cortex and extrastriate areas, corresponding to BA 17, 18, and 19). In both subjects the location of these EEG sources is in agreement with the active "alpha" areas identified by the fMRI analysis when convolving the time course of the EEG alpha map with the hemodynamic response function (HRF). In the second subject (Fig. 3 yellow boxes) we can also observe the localization of secondary sources that are concordant with the BOLD response (Figs. 3.2 and 3.4) in the right parahippocampal gyrus and right fusiform gyrus. We also show projections of the alpha state map into source space (ESI, shown in Figs. 3.5-3.8) using two arbitrary thresholds. As expected ESI accurately computes the location of the sources corresponding to the alpha topography but misses the "alpha network" made of secondary sources temporally highly correlated with the alpha occipital source (as shown in both TESS, Fig. 3.4, and BOLD data, Fig. 3.2). The extent of the sources computed by ESI varies significantly with the arbitrary choice of threshold (Figs. 3.5-3.6 versus Figs. 3.7-3.8).

Furthermore, this dataset showcases the use of TESS for the removal of artifacts typically removed via ICA (eye blink, saccade, cardiac, etc.).

\section{Simulated source imaging}

In Fig. 4 we compare our method accuracy in source localization and amplitude estimation with the accuracy offered by direct projection into source space of the (noisy) map of interest. Such a projection, as well as the noise applied to the original topography, has a combined blurry effect on the final source distribution, as highlighted by the black-andwhite source representation on top of the red box in Fig. 4(B). The application of any ESI method is bound to generate a smooth distribution of sources due to the ill pose-ness of the inverse problem and the need of regularization. Our method, however, benefits from the modified spatial pattern of this intrinsic blurry effect, peaked at the location of the sources, thanks to the spatial distribution of the covariance matrix of $m_{i n v}$ (see Eq. (2)). The source distribution matrix $m_{i n v}$ is computed as the ESI of the EEG measurements, and its covariance matrix is equal to $m_{i n v} \times m_{i n v}^{T}$. As a result, the spatial distribution of the TESS solution $(\hat{\beta})$ 


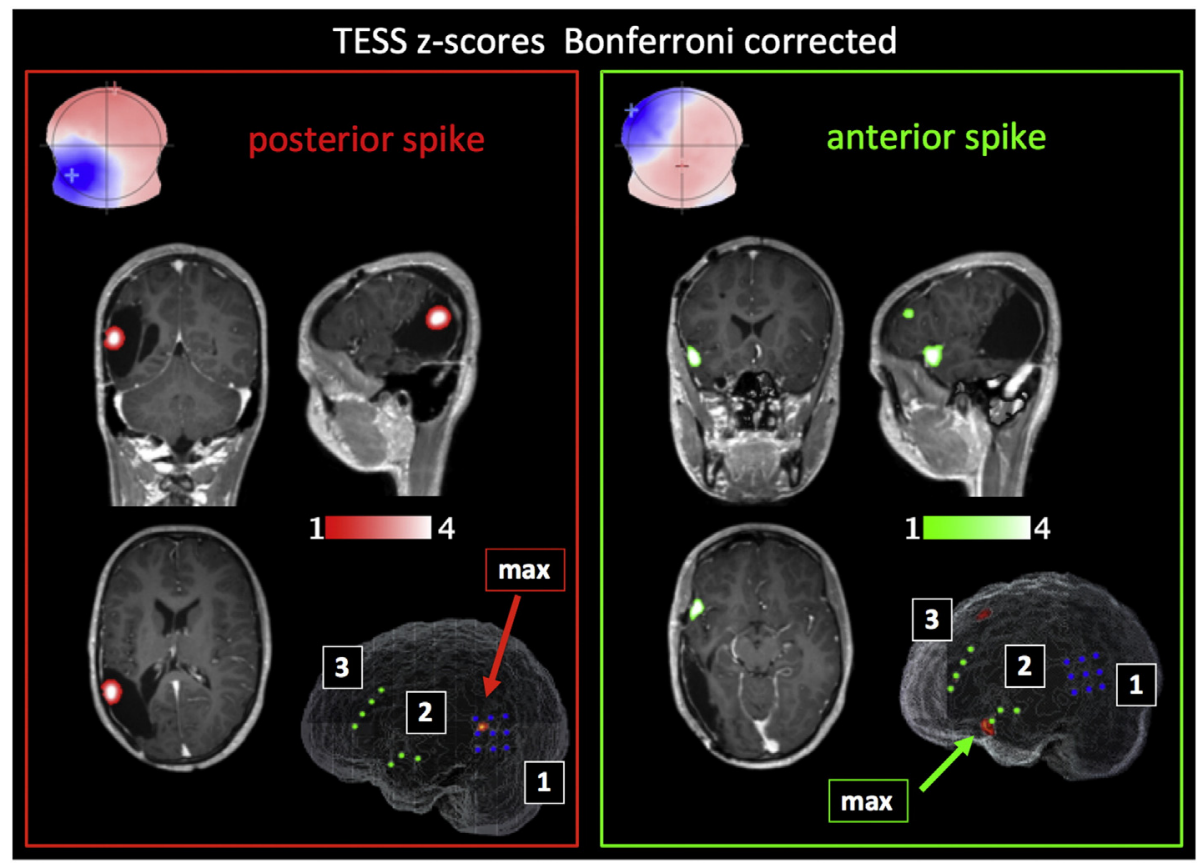

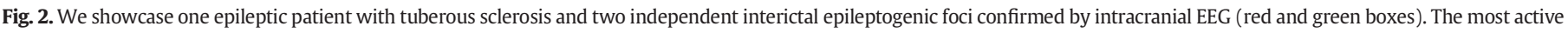

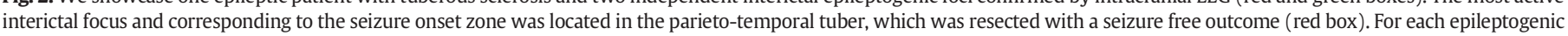

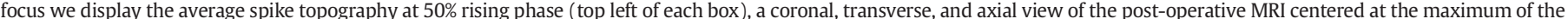

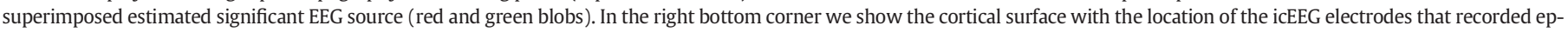

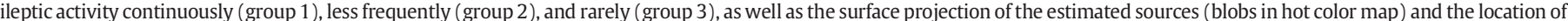

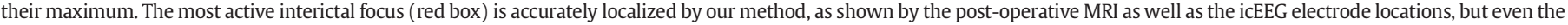
less frequent epileptic activity (green box) was successfully localized, as confirmed by icEEG.

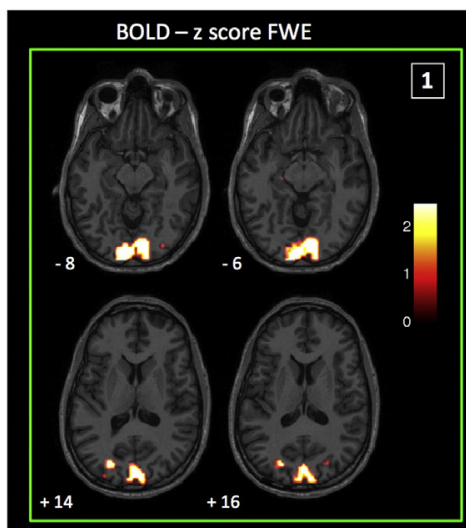

TESS - $\mathrm{z}$ score Bonferroni corrected

ESI of the alpha state map
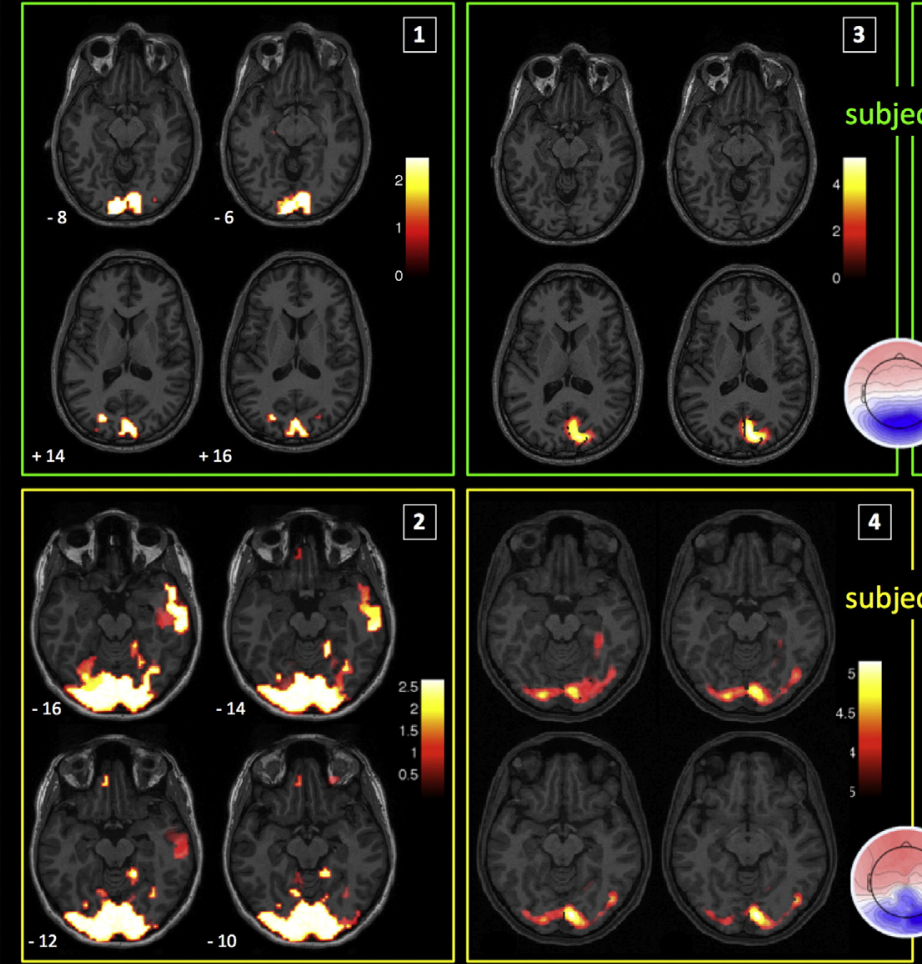

subject 1
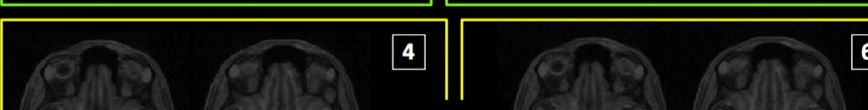

6

subject 2
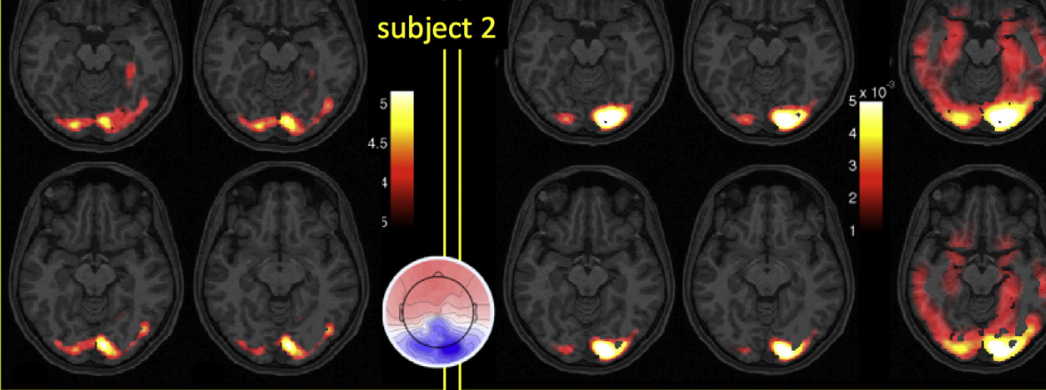

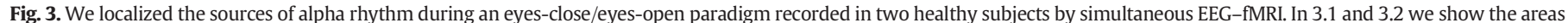

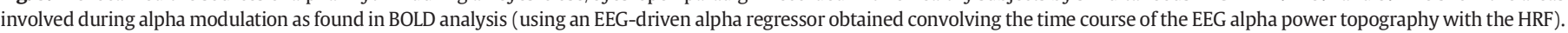

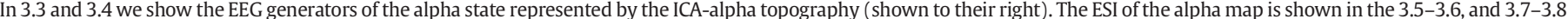

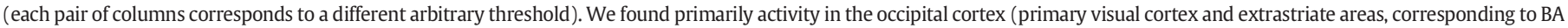

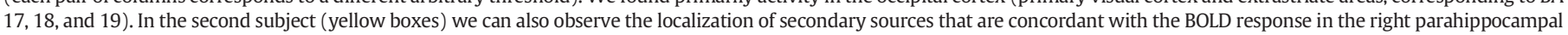
gyrus and right fusiform gyrus. 


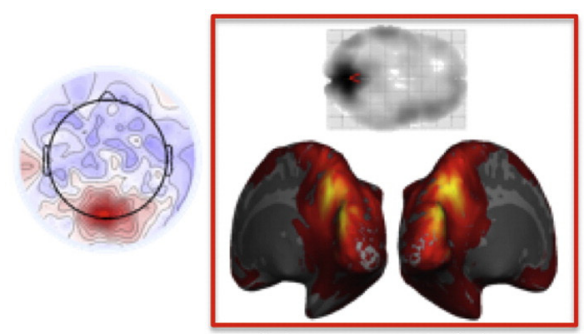

noisy topography (b) and its ESI (B)

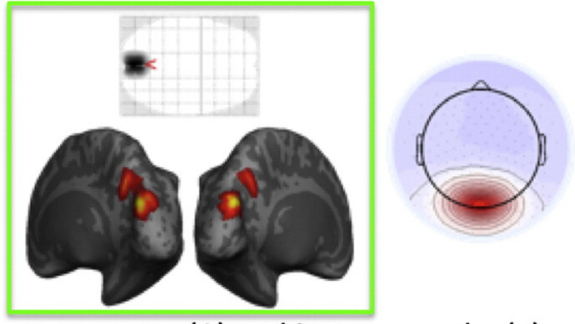

true source (A) and its topography (a)

ROC: $\mathrm{ESI}$ versus $\mathrm{TES}$

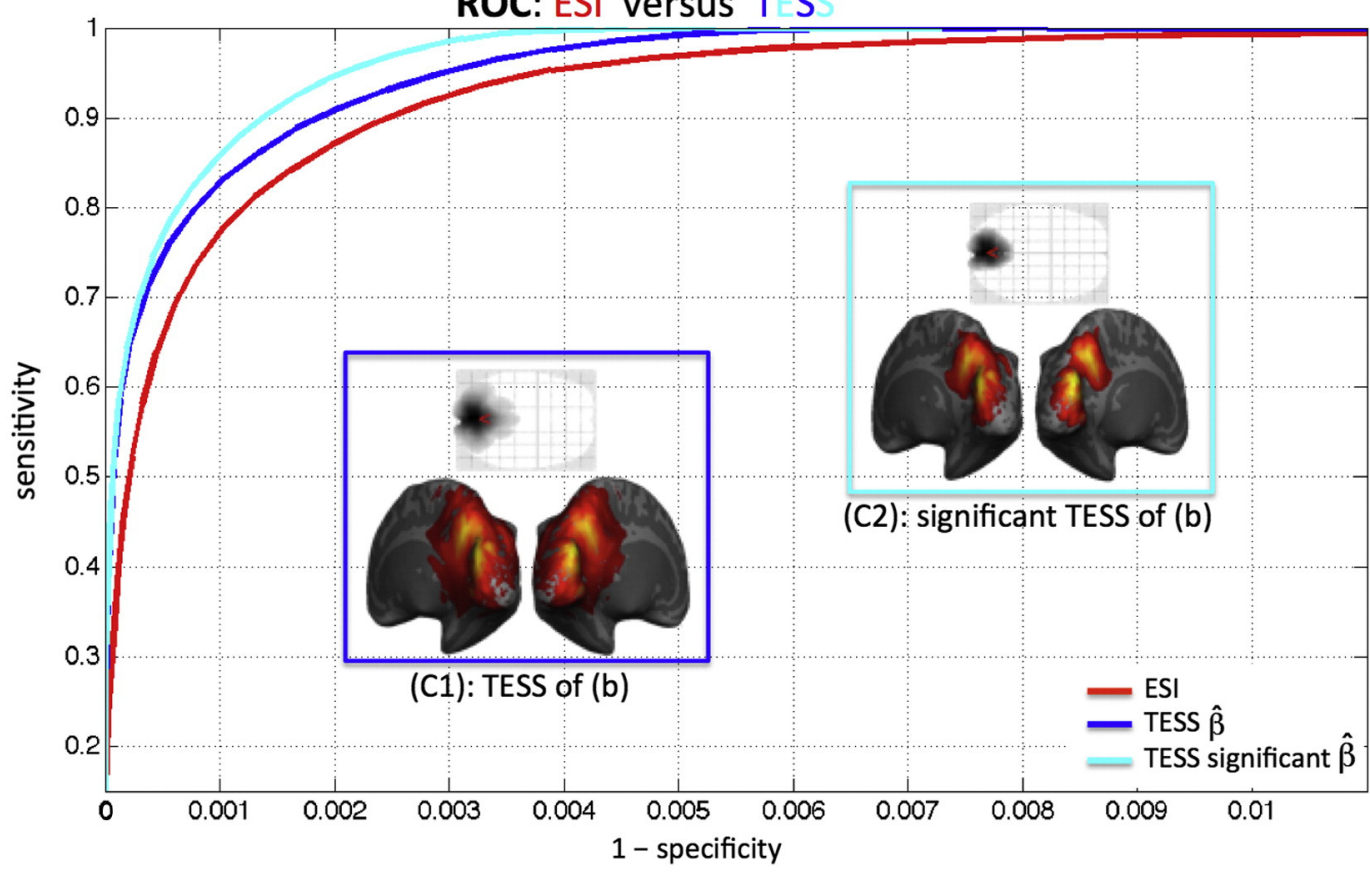

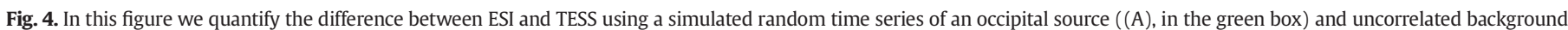

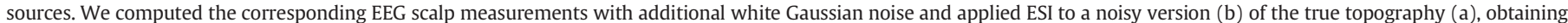

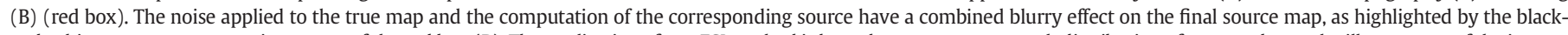

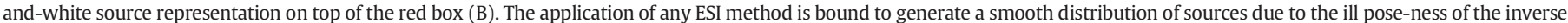

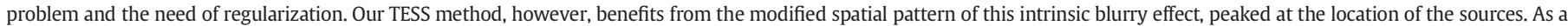

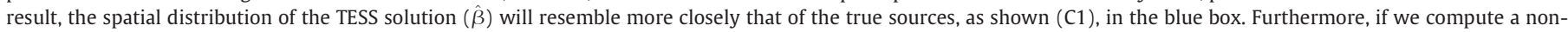

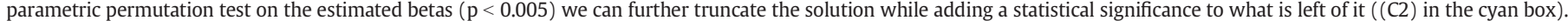

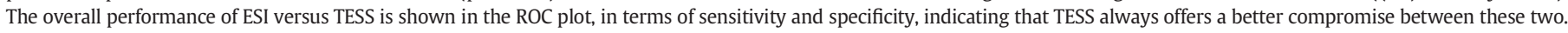

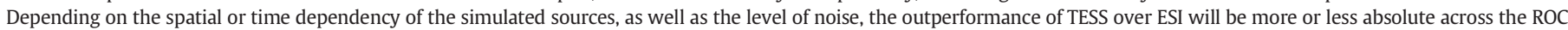
spectrum.

will resemble more closely that of the true sources, as shown in Fig. 4(C1), in the blue box. Even if willing to accept the greater amount of smoothness introduced by an ESI, it will not be feasible to extract state source maps directly in the inverse space because of lack of bijectivity of the inverse operator: a different source map might correspond to the same topography.

Furthermore, if we compute a non-parametric permutation test (see Appendix A) on the estimated betas $(\mathrm{p}<0.005)$ we can further truncate the solution while adding a statistical significance to its remaining distribution (Fig. 4(C2) in the cyan box). The overall performance of ESI versus TESS is shown in the plot of Fig. 4 in terms of sensitivity and specificity (see Appendix A for details), indicating that TESS always offers a better compromise between these two. Depending on the spatial or time dependency of the simulated sources, as well as the level of noise, the outperformance of TESS over ESI will be more or less absolute across the ROC spectrum.

\section{Discussion}

The application of distributed source imaging methods to instantaneous EEG recordings suffers from a severe under-determination of the inverse problem because of the large number of unknown sources in the 3D space and the relatively limited number of measurements (i.e., electrodes). Even when restricting the solution space to the gray matter and fixing the dipole orientation, the number of unknowns largely exceeds the number of sensors that can possibly be applied. To obtain a unique solution of the inverse problem we need to assume a priori constraints. For example, different weighting methods applied to the classical minimum-norm solution (e.g., the Laplacian weights used in LORETA, Pascual-Marqui et al., 1994) provide, in some cases, a reasonable solution to the ill posed problem (Michel et al., 2004). Higher stability against noise is achieved through regularization procedures, but results are becoming spatially blurred. Evaluation of inverse solutions using resolution kernels showed that dipole moments and dipole strengths are systematically underestimated in distributed linear inverse solutions (Grave de Peralta and Gonzalez Andino, 1998). Incorrect estimation of the source strength can lead to ghost sources (Michel et al., 2004) and a low SNR of the recorded EEG can lead to inaccurate source localization. Multiple occurrences of the same event, that assumingly evokes activation of the same sources (e.g., stimulusevoked potentials or multiple epileptic spikes recording), allow a reduction of the effect of low SNR by averaging the data over the repetitions. 
Such repetitions also allow performing statistical analysis in the source space by comparing baseline versus activity periods (Plomp et al., 2010; Sperli et al., 2006). However, we do not always record repetitive events. The most obvious example is the analysis of spontaneous brain activity that, by definition, is not evoked by a repeatedly occurring event. Directly transforming spontaneous activity into the source space using linear inverse solutions and applying signal analysis methods to the estimated source waveforms (De Pasquale et al., 2010) inherently suffers from low SNR, unknown regularization, incorrect strength estimation, creation of ghost sources, etc. (Michel and He, 2011). TESS builds on a chosen ESI method and, therefore, it inherits some of its limitations. However, TESS also improves the accuracy and applicability of the ESI method by estimating the repetitions of the events of interest (expressed by the time courses of the topographies in the state design matrix) and localizing the statistically significant contributing sources; i.e., temporal consistency is exploited maximally.

In the method presented here, we propose to use the temporal dynamics of a given scalp potential map (i.e., the topography of a chosen electrophysiological state) as a posteriori constraint for the inverse solution. There is a close relationship between a scalp potential map and corresponding brain sources: any array of sources in the brain produces a specific configuration of scalp potentials, i.e., a specific topography (Fender, 1987). Consequently, any estimated source waveform in the brain that temporally correlates with the presence of this specific map is likely belonging to the array of sources that produced it. Thus, it is the time course of the source strength systematically correlated with the scalp potential map that counts, and not the instantaneous estimation of the source strength, which, as described above, can be underestimated. Our method is similar to the dual regression described in Beckmann et al. (2009) where fMRI group data is analyzed with a spatial ICA and the resulting components form the equivalent of our state design matrix. Then a spatial GLM estimates the time courses of the independent components (group maps) and a temporal GLM finds the subject-specific maps corresponding to the group-maps.

The described method has been inspired by a similar strategy that was used to analyze the BOLD activity that correlates with certain EEG maps (Britz et al., 2010; Grouiller et al., 2011; Musso et al., 2010). In these studies the temporal dynamics of spontaneous EEG maps or patient-specific epileptic maps have been convolved with the HRF leading to BOLD networks corresponding to the known fMRI resting-state networks or to the known epileptic focus, respectively. These results indicate that the EEG topographies are accurate representations of global brain states, characterized by simultaneous activity of large-scale networks of sources. Our method stems from these EEG-fMRI implementations but with the role of the BOLD data replaced by the EEG source maps. The result is a new EEG state-based source imaging method capable of localizing the generators of electrophysiological states, without being penalized as much by false negatives (lost sources) and false positives (ghost sources) hindering the direct projection of a state map into source space.

TESS' strength hinges on the ability to define a set of state topographies capturing the relevant part of the recorded EEG. If we have relevant accurate topographies, with high SNR, any ESI of these topographies should be sufficient to compute the location, if not directly the extent, of the corresponding sources. However, if we want to investigate the generators of "weak" states (i.e., states with low SNR), TESS is able to estimate the location and extent of the underlying sources, provided the state design matrix is well defined. To this end, when defining the states to include, attention should be given to:

1. Capturing the relevant part of the data: including the state maps corresponding to the predominant events (in amplitude) as well as, if we are interested in studying the more subtle states, the "weak" ones. For example, when recording epileptic spikes, our state design matrix should include secondary state topographies besides the spike maps if we want to analyze effects past the epileptic discharges, like brain networks at rest. Similarly, it is a good practice to add (residual) artifact maps to the design matrix, but it becomes crucial when the artifacts' SNR overcomes the SNR of the targeted states.

2. Different states with near identical time courses will not be distinguishable in source space by TESS; to check for co-linearity between the estimated time courses ( $\hat{T}_{i}$ from Eq. (2), which is $\hat{\beta}_{1}$ in the Appendix's matrix notation) TESS outputs their correlation coefficients and displays a warning if two or more states have highly correlated time courses.

3. If in doubt whether state topographies apparently spatially correlated have distinct generators (small differences in the electrodes space might relate to larger differences in source space, which is a higher dimensional space), one can run TESS with a more inclusive state design matrix and, upon inspecting TESS' output, reduce the matrix as needed and re-compute the results. Typically TESS runs one subject's EEG analysis ( 45,000 time frames, 204 electrodes, 12 maps in the state design matrix, $\sim 5000$ solution points) in a few seconds when skipping the permutation test, or in $\sim 15$ min when performing 1999 permutations.

TESS results can be directly expressed as probability values with a statistical threshold. In the case of epileptic patients, for example, the ESI of an averaged spike map does not provide information on the extent of the epileptic generators, which then depends solely on the arbitrarily chosen threshold of the solution. While statistical post-processing can solve this problem if sufficient spikes are recorded in a given patient (Sperli et al., 2006; Zumsteg et al., 2006), the present method does not require repeated visible occurrences of spikes in the patient's EEG. As already shown by Grouiller et al. (2011), the temporal fluctuation of the epileptic map seems to describe the variations of neuronal activity in the epileptic focus. A reasonable hypothesis is that the significant solution's voxels directly determine the spatial extent of the epileptic zone, similarly to the hypothesis proposed by Chaudhary et al. (2012) using Bayesian methods.

All significant clusters identify sources time-locked with the state represented by a given topography (e.g., the spike map, in Fig. 2). Although for the epileptic data we focus on the cluster with maximal activity, conforming to the clinical use of other EEG- or fMRI-based localizing techniques (Brodbeck et al., 2011; Grouiller et al., 2011), the neurophysiological significance of secondary clusters in terms of involvement in neuronal networks modulating epileptic activity is highly relevant and interesting, but it is not the scope of this paper. Secondary clusters might indeed be true positives when considering not only the epileptogenic network but also the localization of a putative focus amenable to surgery (Richardson, 2012). A global analysis of TESS results considering all statistically significant (secondary) clusters is certainly important for better understanding the benefits and shortcomings of this method but requires a separate study with a larger group of patients and additional source-localization tools serving as ground truth.

An important application of our method is the localization of brain networks that participate in the generation of meaningful rhythms in the EEG, like the alpha rhythm highlighted in this paper. Many simultaneous EEG-fMRI studies convolve the time-course of the EEG power of selected electrodes, or the sum of all electrodes at a selected frequency band, with the HRF to compute the BOLD correlates to EEG selected frequencies (Laufs et al., 2003; Mantini et al., 2007; Tyvaert et al., 2008). This approach ignores the fact that different scalp potential maps exist for any given frequency with different time-courses (Koenig et al., 2001), directly indicating that in the brain there are different generators working independently in the same frequency. Using time-frequency decomposition as proposed in Koenig et al. (2001) we can identify all the frequency-specific maps and our method can use these topographies to compute the complete set of independent generators contributing to the same EEG rhythm.

Another consideration to be made is that EEG at rest can be analyzed with this procedure in a similar fashion as demonstrated in previous 
EEG-fMRI studies (Britz et al., 2010; Musso et al., 2010). The low temporal correlation, or even anti-correlation, of the resting state EEG maps makes them ideal candidates to separate the corresponding sources using the proposed approach. Since our method generates statistical maps for each subject, we can quantify stability of the resting state networks within and across subjects as done for fMRI resting state maps. Since many studies have demonstrated a relationship between the temporal dynamics of resting EEG maps and different mental diseases (Nishida et al., 2013), our method is particularly suited to further our understanding of psychiatric disorders non-invasively.

\section{Conclusions}

Unlike conventional EEG source imaging method, our spatiotemporal regression method can differentiate between electrophysiological sources of interest and linearly independent background sources (neurophysiological or not) in a single convenient framework. Once the EEG traces are analyzed and decomposed into topographically stable states (using ICA, k-means, epoch average, etc.), the method will compute the statistically significant generators of each defined state. The main features of the proposed method are:

1. The set of states driving the method can be generated from the EEG data itself or computed otherwise (group maps, suspected epileptic generators without spike recording, simulated maps, etc.).

2. The method is robust against most common artifacts: adding nonphysiological topographies to the method's set of states will have the same effect as an ICA back-projection, with the additional advantage that the artifact maps can be simulated avoiding any further EEG pre-processing step.

3. Similarly to what has been proposed for fMRI analysis (Chaudhary et al., 2012), it is possible to add maps to the model as cofounds to capture the residual unexplained part of the data (e.g., for restmicrostate analysis in epileptic patients: combining epileptic topographies with rest-state topographies; in evoke potential analysis: adding rest-microstates and predominant rhythm topographies helps separate linearly coupled baseline effects from true evoked responses).

4. While using state topographies, our method has proven effective in accurately estimating the time course of spontaneous activity (alpha rhythm, see Fig. 3) and correctly identifying the corresponding significant generators in the brain. The ability to find and statistically threshold the sources of EEG spontaneous activity promises further insight in rest-microstates analysis and its application to early diagnosis of mental diseases (Lehmann et al., 2005).

5. Our method makes use of an Electrical Source Imaging method (any ESI method) and it inherits the chosen ESI method's strengths as well as biases (e.g.: cortical regions affected by a lower SNR, artifacts due to simplified head models (Montes-Restrepo et al., 2014), inaccurate electrodes' coregistration, inadequate electrodes' number or distribution, forward model's errors (Akalin Acar and Makeig, 2013), etc.).

Future work includes several applications of our method to eventrelated and spontaneous activity measurements. In one hand we believe that we will be able to shed new light into rest-microstates source analysis, in the other we expect better source localization and source amplitude estimation by using our method in better known cases (e.g., epilepsy and evoked potentials), also by adding background activity and non-physiological activity maps to our state model.

\section{Acknowledgments}

This work was supported by the Swiss National Science Foundation through the National Center of Competence in Research (NCCR) "SYNAPSY-The Synaptic Basis of Mental Diseases" as well as the research grants No. 310030_132952 to C.M.M., No. PP00P2-146318 to DVDV., and No. 141165 to S.V. Additional support was granted by the Center for Biomedical Imaging (CIBM), Geneva and Lausanne, Switzerland.

\section{Appendix A}

Method

Here we derive the full matrix expression of the consecutive fitting by the spatial and temporal GLMs as applied in TESS. We define $V$ as the number of solution points in source space, $E$ as the number of sensors, $T$ as the number of time points, and $S$ as the number of state topographies in the design matrix $M_{e}: E \times S$. The projection operator $A: E \times V$ incorporates the forward model from source to sensor space, and $B: V \times E$ is the inverse solution operator from sensor space to source space. Further on, $X_{e}: E \times T$ is the EEG data in sensor space.

The different steps can then be described as follows:

1. (Spatial) GLM I:

Putting forward the GLM $X_{e}=M_{e} \cdot \beta_{1}$, we derive the estimate of $\beta_{1}$ coefficients as:

$\hat{\beta}_{1}=M_{e}^{\dagger} \cdot X_{e}: S \times T$,

where $(\ldots)^{\dagger}$ is the notation for the pseudo-inverse operator; i.e., $A^{\dagger}=$ $\left(A^{T} A\right)^{-1} A^{T}$.

2. Source-space projection, which leads to $X_{s}: V \times T$, the reconstructed data in source space for each time instance:

$X_{s}=B \cdot X_{e}: V \times T$.

3. (Temporal) GLM II:

Putting forward the GLM $X_{s}^{T}=\hat{\beta}_{1}^{T} \cdot \beta_{2}$, we derive the estimate of $\beta_{2}$ coefficients as:

$\hat{\beta}_{2}=\left(\hat{\beta}_{1}^{T}\right)^{\dagger} \cdot\left(X_{S}\right)^{T}: S \times V$

from which we obtain: $\hat{\beta}_{2}=\left(X_{e}^{T} \cdot M_{e}^{\dagger^{T}}\right)^{\dagger} \cdot X_{e}^{T} \cdot B^{T}$. We can now expand the expression of the outer pseudo-inverse, from which $\hat{\beta}_{2}$ can be rewritten as:

$\hat{\beta}_{2}=\left[\left(X_{e}^{T} \cdot M_{e}^{\dagger^{T}}\right)^{T} \cdot\left(X_{e}^{T} \cdot M_{e}^{\dagger^{T}}\right)\right]^{-1} \cdot\left(X_{e}^{T} \cdot M_{e}^{\dagger^{T}}\right)^{T} \cdot X_{e}^{T} \cdot B^{T}$,

applying the outer transpose, it becomes:

$\hat{\beta}_{2}=\left[M_{e}^{\dagger} \cdot X_{e} \cdot X_{e}^{T} \cdot M_{e}^{\dagger^{T}}\right]^{-1} \cdot M_{e}^{\dagger} \cdot X_{e} \cdot X_{e}^{T} \cdot B^{T}$.

From the definition of covariance matrix in sensor space: $C_{e}=X_{e} \cdot X_{e}^{T}$, we obtain:

$$
\begin{aligned}
\hat{\beta}_{2} & =\left[M_{e}^{\dagger} \cdot C_{e} \cdot M_{e}^{\dagger^{T}}\right]^{-1} \cdot M_{e}^{\dagger} \cdot C_{e} \cdot B^{T} \\
& =\left[M_{e}^{\dagger} \cdot C_{e}^{1 / 2} \cdot C_{e}^{1 / 2} \cdot M_{e}^{\dagger^{T}}\right]^{-1} \cdot M_{e}^{\dagger} \cdot C_{e}^{1 / 2} \cdot C_{e}^{1 / 2} \cdot B^{T},
\end{aligned}
$$

where we have factorized the covariance matrix (a positivesemidefinite matrix, by construction) using its principal square root. Taking the transpose of the transpose of $M_{e}^{\dagger} \cdot C_{e}^{1 / 2}$ and noting that $C_{e}^{1 / 2}=C_{e}^{1 / 2^{T}}$, we derive:

$\hat{\beta}_{2}=\left[\left(C_{e}^{1 / 2^{T}} \cdot M_{e}^{\dagger^{T}}\right)^{T} \cdot\left(C_{e}^{1 / 2^{T}} \cdot M_{e}^{\dagger^{T}}\right)\right]^{-1} \cdot\left(C_{e}^{1 / 2^{T}} \cdot M_{e}^{\dagger^{T}}\right)^{T} \cdot C_{e}^{1 / 2} \cdot B^{T}$,

given that: $A^{\dagger}=\left(A^{T} A\right)^{-1} A^{T}$, we rewrite the equation as:

$\hat{\beta}_{2}=\left(C_{e}^{1 / 2} \cdot M_{e}^{\dagger^{T}}\right)^{\dagger} \cdot C_{e}^{1 / 2} \cdot B^{T}$. 


\section{Non-parametric statistical testing}

To evaluate the statistical significance of the fitted coefficients in the solution space (obtained after the 2nd GLM), we performed a nonparametric permutation test since we observed that their distribution is non-Gaussian (Nichols and Holmes, 2002; Winkler et al., 2014). In particular, we applied Fourier phase randomization on $\hat{\beta}_{1}$ (number of state maps $\times$ time points) to obtain surrogate regressors; the same phase randomization is applied to each regressor (Bullmore et al., 2001; Theiler et al., 1992). However, for the alpha-rhythm validation experiment, we used temporal permutation of blocks with length $100 \mathrm{~ms}$, because phase randomization did not allow for sufficient randomness of the surrogate regressors with respect to the true ones. Then, we fitted source maps using these surrogate temporal regressors. We repeated the procedure 1999 times, which effectively generates an empirical null-distribution of the source-maps (smallest possible $\mathrm{p}=0.0005)$. Finally, we use this empirical distribution to compute equivalent $\mathrm{z}$-scores, in particular, in the tail of the distribution $(0.0005<\mathrm{p}<0.005)$. The $\mathrm{z}$-scores were Bonferroni corrected to account for multiple comparisons by combining the null-distributions of all solution points (4000-5000 points). We used different correction methods (i.e., Gauss-random Fields theory FWE and Bonferroni correction for BOLD and TESS, respectively) considering the number of observations in each statistical test (in the order of 800,000-900,000 for BOLD data and 4000-5000 for TESS). We opted for this choice since applying Bonferroni for BOLD results would be clearly over-conservative, while FWE for TESS would require non-trivial adaptation of Gaussian random field theory to the (non-Cartesian) grid of solution points.

\section{Simulation parameters}

\section{Simulated EEG}

We simulated an EEG with 204 electrodes and 6000 time points. The EEG was defined as $X_{e}=B \cdot X_{s}$, i.e.: the projection into sensors space (B) of $X_{s}$, where $X_{S}=M_{S} \cdot T_{s}$ is the simulated brain activity made of 3 sourcemaps: $M_{s}=\left[\begin{array}{lll}m_{s 1} & m_{s 2} & m_{s 3}\end{array}\right]$, with time courses $T_{s}=\left[\begin{array}{c}t_{s 1} \\ t_{s 2} \\ t_{s 3}\end{array}\right]$ :

$m_{s 1} \quad$ is the background noise map; it is continuously present with $t_{s 1}=K$, where $K$ is a [ $\left.1 \times k\right]$ matrix drawn from a Gaussian distribution, $\mathcal{N}(0,1)$, and $k=$ number of time points and $m_{s 1}=W \cdot 0.01$, where $W$ is a $[m \times 1]$ matrix also drawn from a Gaussian distribution, $\mathcal{N}(0,1)$, and $m=$ number of solution points.

$m_{s 2} \quad$ is the confound map: $m_{s 2}=W \cdot 0.1$ and it is present at random times (on/off) with random durations.

$m_{s 3} \quad$ is the occipital map, the source-map of interest; it is a normalized simulated occipital source with radius $5 \mathrm{~mm}$ and direction along the norm to the cortical surface at its centroid location; this map is present at random times (on/off) with random durations.

In the sensor space we define: $m_{e 2}=A \cdot W, m_{e 3}=A \cdot m_{s 3}, Q$ is a $[n \times 1]$ matrix s.t. $Q \in \mathcal{N}(0,1), n=$ number of electrodes, and $A: E \times V$ is the projection operator from source space to sensor space. TESS regressors are $m_{e 2} \cdot 0.01+Q$ and $m_{e 3} \cdot 0.1+Q$. In Fig. 4 we show the true map $m_{e 3}$ (Fig. 4a) and the perturbed topography $m_{m 3} \cdot 0.1+Q$ (Fig. 4b).

\section{$R O C$}

Sensitivity and specificity are computed using the standard definitions: sensitivity $=\mathrm{TP} /(\mathrm{TP}+\mathrm{FN})$, and specificity $=\mathrm{TN} /(\mathrm{TN}+\mathrm{FP})$; where TP/TN/FP/FN (true positives/true negatives/false positives/false negatives) are computed as the difference between the number of binarized active/non-active voxels in the estimated source map (ESI or TESS) and the ground truth ( $m_{s 3}$, i.e.: the simulated occipital source). For the computation of the number of true/false positives/negatives, as well as TESS's results graphical display, we interpolated that the recovered signal on the EEG solution points to the 3-D Cartesian brain volume using heat diffusion with a Gaussian kernel (sigma of $2.5 \mathrm{~mm}$ and 10 iterations) and fixing the EEG solution points as boundary conditions.

\section{References}

Akalin Acar, Z., Makeig, S., 2013. Effects of forward model errors on EEG source localization. Brain Topogr. 26 (3), 378-396.

Allen, P.J., Polizzi, G., Krakow, K., Fish, D.R., Lemieux, L., 1998. Identification of EEG events in the MR scanner: the problem of pulse artifact and a method for its subtraction. Neuroimage 8, 229-239.

Allen, P.J., Josephs, O., Turner, R.A., 2000. Method for removing imaging artifact from continuous EEG recorded during functional MRI. Neuroimage 12, 230-239.

Bandettini, P.A., Wong, E.C., Hinks, R.S., Tikofsky, R.S., Hyde, J.S., 1992. Time course EPI of human brain function during task activation. Magn. Reson. Med. 25, 390-397.

Beckmann, C.F., Mackay, C.E., Filippini, N., Smith, S.M., 2009. Group comparison of restingstate FMRI data using multi-subject ICA and dual regression. NeuroimageAcademic Press.

Bell, A.J., Sejnowski, T.J., 1995. An information-maximization approach to blind separation and blind deconvolution. Neural Comput. 7, 1129-1159.

Brandeis, D., Lehmann, D., 1986. Event-related potentials of the brain and cognitive processes: approaches and applications. Neuropsychologia 24 (1), 151-168.

Britz, J., Van De Ville, D., Michel, C.M., 2010. BOLD correlates of EEG topography reveal rapid resting-state network dynamics. Neuroimage 52, 1162-1170.

Brodbeck, V., Spinelli, L., Lascano, A.M., Wissmeier, M., Vargas, M.I., Vulliemoz, S., Pollo, C. Schaller, K., Michel, C.M., Seeck, M., 2011. Electroencephalographic source imaging: a prospective study of 152 operated epileptic patients. Brain 134, 2887-2897.

Brookes, M., Gibson, A., Hall, S., Furlong, P., Barnes, G., Hillebrand, A., Singh, K., Halliday, I., Francis, S., Morris, P., 2004. A general linear model for MEG beamformer imaging. NeuroImage 23 (3), 936-946.

Brunet, D., Murray, M.M., Michel, C.M., 2011. Spatiotemporal analysis of multichannel EEG: CARTOOL. Comput. Intell. Neurosci. 2011, 813870

Bullmore, E., Long, C., Suckling, J., Fadili, J., Calvert, G., Zelaya, F., Carpenter, T.A., Brammer, M., 2001. Colored noise and computational inference in neurophysiological (fMRI) time series analysis: resampling methods in time and wavelet domains. Hum. Brain Mapp. 12 (2), 61-78.

Chaudhary, U.J., Rodionov, R., Carmichael, D.W., Thornton, R.C., Duncan, J.S., Lemieux, L., 2012. Improving the sensitivity of EEG-fMRI studies of epileptic activity by modeling eye blinks, swallowing and other video-EEG detected physiological confounds. Neuroimage 61 (4), 1383-1393.

De Lucia, M., Michel, C.M., Clarke, S., Murray, M.M., 2007. Single-trial topographic analysis of human EEG: a new 'image' of event-related potentials. Proceedings Information Technology Applications in Biomedicine. ITAB, pp. 95-98 (article 4407353).

De Martino, F., Valente, G., de Borst, A.W., Esposito, F., Roebroeck, A., Goebel, R., Formisano, E., 2010. Multimodal imaging: an evaluation of univariate and multivariate methods for simultaneous EEG/fMRI. Magn. Reson. Imaging 28, 1104-1112.

De Pasquale, F., Della Penna, S., Snyder, A.Z., Lewis, C., Mantini, D., Marzetti, L., Belardinelli, P., Ciancetta, L., Pizzella, V., Romani, G.L., Corbetta, M., 2010. Temporal dynamics of spontaneous MEG activity in brain networks. Proc. Natl. Acad. Sci. U. S. A. 107 (13), $6040-6045$

Debener, S., Mullinger, K.J., Niazy, R.K., Bowtell, R.W., 2008. Properties of the ballistocardiogram artefact as revealed by EEG recordings at 1.5, 3 and $7 \mathrm{~T}$ static magnetic field strength. Int. J. Psychophysiol. 67 (3), 189-199 (Epub 2007 Jul 12. PubMed PMID: 17683819).

Fender, D.H., 1987. Source localization of brain electrical activity. In: Gevins, A.S., Remond, A. (Eds.), Handbook of Electroencephalography and Clinical Neurophysiology. Methods of Analysis of Brain Electrical and Magnetic Signals, vol. 1. Elsevier, Amsterdam, pp. 355-399.

Friston, K.J., Holmes, A.P., Worsley, K.J., Poline, J.B., Frith, C., Frackowiak, R.S.J., 1995. Statistical parametric maps in functional imaging: a general linear approach. Hum. Brain Mapp. 2, 189-210.

Grave de Peralta, M.R., Gonzalez Andino, S.L., 1998. A critical analysis of linear inverse solutions. IEEE Trans. Biomed. Eng. 45, 440-448.

Grave de Peralta, M.R., Murray, M.M., Michel, C.M., Martuzzi, R., Gonzalez Andino, S.L, 2004. Electrical neuroimaging based on biophysical constraints. Neuroimage 21 (2), 527-539.

Grouiller, F., Thornton, R.C., Groening, K., Spinelli, L., Duncan, J.S., Schaller, K., Siniatchkin, M., Lemieux, L., Seeck, M., Michel, C.M., Vulliemoz, S., 2011. With or without spikes: localization of focal epileptic activity by simultaneous electroencephalography and functional magnetic resonance imaging. Brain 134, 2867-2886.

Jung, T.P., Makeig, S., McKeown, M.J., Bell, A.J., Lee, T.W., Sejnowski, T.J., 2001. Imaging brain dynamics using independent component analysis. Proc. IEEE Inst. Electr. Electr. Eng. 89 (7), 1107-1122.

Kargiotis, O., Lascano, A.M., Garibotto, V., Spinelli, Lb, Genetti, M., Wissmeyer, M., Korff, C. M., Momjian, S., Michel, C.M., Seeck, M., Vulliemoz, S., 2014 Feb. Localization of the epileptogenic tuber with electric source imaging, in patients with tuberous sclerosis. Epilepsy Res. 108 (2), 267-279. http://dx.doi.org/10.1016/j.eplepsyres.2013.11.003.

Kiebel, S.J. Friston, KJ. 2004. Statistical parametric mapping for event-related potentials: II. A hierarchical temporal model. Neurolmage 22 (2), 503-520. 
Kikuchi, M., Koenig, T., Wada, Y., Higashima, M., Koshino, Y., Strik, W., Dierks, T., 2007. Native EEG and treatment effects in neuroleptic-naïve schizophrenic patients: time and frequency domain approaches. Schizophr. Res. 97 (1-3), 163-172.

Koenig, T., Marti-Lopez, F., Valdes-Sosa, P., 2001. Topographic time-frequency decomposition of the EEG. Neuroimage 14 (2), 383-390.

Kwong, K.K., Belliveau, J.W., Chesler, D.A., Goldberg, I.E., Weisskoff, R.M., Poncelet, B.P., Kennedy, D.N., Hoppel, B.E., Cohen, M.S., Turner, R., 1992. Dynamic magnetic resonance imaging of human brain activity during primary sensory stimulation. Proc. Natl. Acad. Sci. U. S. A. 89, 5675-5679.

Lantz, G., Spinelli, L., Seeck, M., de Peralta Menendez, R.G., Sottas, C.C., Michel, C.M., 2003. Propagation of interictal epileptiform activity can lead to erroneous source localizations: a 128-channel EEG mapping study. J. Clin. Neurophysiol. 20 (5), 311-319.

Laufs, H., Kleinschmidt, A., Beyerle, A., Eger, E., Salek-Haddadi, A., Preibisch, C., Krakow, K., 2003. EEG-correlated fMRI of human alpha activity. Neuroimage 19 (4), 1463-1476

Lehmann, D., Strik, W.K., Henggeler, B., Koenig, T., Koukkou, M., 1998. Brain electric microstates and momentary conscious mind states as building blocks of spontaneous thinking: I. Visual imagery and abstract thoughts. Int. J. Psychophysiol. 29, 1-11.

Lehmann, D., Faber, P.L., Galderisi, S., Herrmann, W.M., Kinoshita, T., Koukkou, M., Mucci, A., Pascual-Marqui, R.D., Saito, N., Wackermann, J., Winterer, G., Koenig, T., 2005. EEG microstate duration and syntax in acute, medication-naive, first-episode schizophrenia: a multi-center study. Psychiatry Res. 138 (2), 141-156.

Lehmann, D., Pascual-Marqui, R., Michel, C.M., 2009. EEG microstates. Scholarpedia 4, 7632.

Makeig, S., Jung, T.P., Bell, A.J., Ghahremani, D., Sejnowski, T.J., 1997. Blind separation of auditory event-related brain responses into independent components. Proc. Natl. Acad. Sci. U. S. A. 94 (20), 10979-10984.

Mantini, D., Perrucci, M.G., Del Gratta, C., Romani, G.L., Corbetta, M., 2007. Electrophysiological signatures of resting state networks in the human brain. Proc. Natl. Acad. Sci. U. S. A. 104 (32), 13170-13175.

Mégevand, P., Spinelli, L., Genetti, M., Brodbeck, V., Momjian, S., Schaller, K., Michel, C.M., Vulliemoz, S., Seeck, M., 2014 Jan. Electric source imaging of interictal activity accurately localises the seizure onset zone. J. Neurol. Neurosurg. Psychiatry 85 (1), 38-43. http://dx.doi.org/10.1136/jnnp-2013-305515.

Michel, C.M., He, B., 2011. EEG mapping and source imaging. In: Schomer, D., Lopes daSilva, F.H. (Eds.), Niedermeyer's Electroencephalography. Lippincott Williams \& Wilkins, Philadelphia, pp. 1179-1202.

Michel, C.M., Murray, M.M., 2012. Towards the utilization of EEG as a brain imaging tool. Neuroimage 61 (2), 371-385.

Michel, C.M., Grave de Peralta, R., Lantz, G., Gonzalez Andino, S., Spinelli, L., Blanke, O., Landis, T., Seeck, M., 1999. Spatiotemporal EEG analysis and distributed source estimation in presurgical epilepsy evaluation. J. Clin. Neurophysiol. 16 (3), 239-266 (Review).

Michel, C.M., Thut, G., Morand, S., Khateb, A., Pegna, A.J., Grave de Peralta, R., Gonzalez, S., Seeck, M., Landis, T., 2001. Electric source imaging of human brain functions. Brain Res. Rev. 36 (2-3), 108-118.

Michel, C.M., Murray, M.M., Lantz, G., Gonzalez, S., Spinelli, L., Grave de Peralta, R., 2004. EEG source imaging. Clin. Neurophysiol. 115, 2195-2222.

Montes-Restrepo, V., van Mierlo, P., Strobbe, G., Staelens, S., Vandenberghe, S., Hallez, H., 2014 Jan. Influence of skull modeling approaches on EEG source localization. Brain Topogr. 27 (1), 95-111. http://dx.doi.org/10.1007/s10548-013-0313-y.
Musso, F., Brinkmeyer, J., Mobascher, A., Warbrick, T., Winterer, G., 2010. Spontaneous brain activity and EEG microstates. A novel EEG/fMRI analysis approach to explore resting-state networks. Neuroimage 52 (4), 1149-1161.

Nichols, T.E., Holmes, A.P., 2002. Nonparametric permutation tests for functional neuroimaging: a primer with examples. Hum. Brain Mapp. 15 (1), 1-25.

Nishida, K., Morishima, Y., Yoshimura, M., Isotani, T., Irisawa, S., Jann, K., Dierks, T., Strik W., Kinoshita, T., Koenig, T., 2013. EEG microstates associated with salience and frontoparietal networks in frontotemporal dementia, schizophrenia and Alzheimer's disease. Clin. Neurophysiol. 124 (6), 1106-1114.

Ogawa, S., Tank, D.W., Menon, R., Ellermann, J.M., Kim, S.G., Merkle, H., Ugurbil, K., 1992. Intrinsic signal changes accompanying sensory stimulation: functional brain mapping with magnetic resonance imaging. Proc. Natl. Acad. Sci. U. S. A. 89, 5951-5955.

Onton, J., Westerfield, M., Townsend, J., Makeig, S., 2006. Imaging human EEG dynamics using independent component analysis. Neurosci. Biobehav. Rev. 30 (6), 808-822.

Pascual-Marqui, R.D., Michel, C.M., Lehmann, D., 1994. Low resolution electromagnetic tomography: a new method for localizing electrical activity in the brain. Int. J. Psychophysiol. 18 (1), 49-65.

Pascual-Marqui, R.D., Michel, C.M., Lehmann, D., 1995. Segmentation of brain electrical activity into microstates: model estimation and validation. IEEE Trans. Biomed. Eng. 42 658-665.

Plomp, G., Michel, C.M., Herzog, M.H., 2010. Electrical source dynamics in three functional localizer paradigms. Neuroimage 53 (1), 257-267.

Richardson, M.P., 2012. Large scale brain models of epilepsy: dynamics meets connectomics. J. Neurol. Neurosurg. Psychiatry 83 (12), 1238-1248. http://dx.doi. org/10.1136/jnnp-2011-301944 (Dec).

Rihs, T.A., Michel, C.M., Thut, G., 2009. A bias for posterior alpha-band power suppression versus enhancement during shifting versus maintenance of spatial attention. Neuroimage 44, 190-199.

Scherg, M., Ille, N., Weckesser, D., Ebert, A., Ostendorf, A., Boppel, T., Schubert, S., Larsson, P.G., Henning, O., Bast, T., 2012. Fast evaluation of interictal spikes in long-term EEG by hyper-clustering. Epilepsia 53 (7), 1196-1204.

Sperli, F., Spinelli, L., Seeck, M., Kurian, M., Michel, C.M., Lantz, G., 2006. EEG source imaging in pediatric epilepsy surgery: a new perspective in presurgical workup. Epilepsia 47 (6), 981-990.

Theiler, J., Eubank, S., Longtin, A., Galdrikian, B., Doyne Farmer, J., 1992. Testing for nonlinearity in time series: the method of surrogate data. Phys. D 58 (1), 77-94.

Trujillo-Barreto, N.J., Aubert-Vázquez, E., Penny, W.D., 2008. Bayesian M/EEG source reconstruction with spatio-temporal priors. Neuroimage 39 (1), 318-335 (Epub 2007 Aug 22).

Tyvaert, L., Levan, P., Grova, C., Dubeau, F., Gotman, J., 2008. Effects of fluctuating physiological rhythms during prolonged EEG-fMRI studies. Clin. Neurophysiol. 119 (12), 2762-2774.

Winkler, A.M., Ridgway, G.R., Webster, M.A., Smith, S.M., Nichols, T.E, 2014. Permutation inference for the general linear model. Neurolmage 92C, 381-397 (doi: 10.1016).

Zelmann, R., Lina, J.M., Schulze-Bonhage, A., Gotman, J., Jacobs, J., 2013 Oct 19. Scalp EEG is not a blur: it can see high frequency oscillations although their generators are small. Brain Topogr. [Epub ahead of print] PubMed PMID: 24141890.

Zumsteg, D., Friedman, A., Wieser, H.G., Wennberg, R.A., 2006. Source localization of interictal epileptiform discharges: comparison of three different techniques to improve signal to noise ratio. Clin. Neurophysiol. 117 (3), 562-571. 